\title{
Acontecimientos vitales estresantes en la infancia y adolescencia de personas sentenciadas en España
}

\author{
Stressful vital events in the infancy and adolescence \\ of sentenced people in Spain
}

\author{
Cristina Estrada-Pineda \\ Francisco Javier Rodríguez-Díaz \\ Carolina Bringas-Molleda \\ Mario Alberto Morales-Martínez \\ Centro Universitario de Ciencias Sociales y Humanidades de la Universidad \\ de Guadalajara, México. \\ Departamento de Psicología de la Universidad de Oviedo, España \\ Universidad de Extremadura, España \\ Centro Universitario del Norte de la Universidad de Guadalajara, México
}

Resumen

Es un estudio cualitativo de registros autobiográficos de internos sentenciados por algún delito en un Centro Penitenciario de España; se estudian 23 historias mediante el análisis de narrativas para indagar las experiencias vividas de los Acontecimientos Vitales Estresantes, recurrentes en esta población, y cómo se van entrelazando en su desarrollo de vida; estos hechos son considerados sucesos que impactan la vida, e incluso, la identidad de los individuos (Sanz Rodríguez et al., 2009). Los resultados permitieron configurar un mapa donde destacan tres grandes categorías recurrentes de AVE en las autobiografías, integradas cada una por dos elementos afines: presencia de adicciones y delito, trayectoria escolar y estilo educativo/relacional, y por último, violencia intrafamiliar y ruptura de pareja.

Palabras clave: Delincuencia, acontecimientos vitales estresantes, autobiografías, infancia y adolescencia.

Abstract

It is a qualitative study of autobiographical registers of sentenced interns of a crime in a Spain Penitentiary Center; 23 stories are studied through the analysis of the narratives to investigate the lived experiences of the Stressful Vital Events, recurrent in this population, and how they are interrelated in the development of their lives; these events are considered events that impact life, including the identity of individuals (Sanz Rodríguez et al., 2009). The results allowed the configuration of a map where three big recurrent categories of the Stressful Vital Events of the autobiographies stand out, each integrated by two related elements: presence of school trajectory and educational/relational style, addictions and crime, and finally, intrafamilial violence and couple rupture.

Key words: Delinquency, stressful vital events, autobiographies, infancy and adolescence. 


\section{INTRODUCCIÓN}

$\mathbf{E}$ 1 estudio de las causas que llevan a los individuos a cometer algún delito tiene un extenso desarrollo actualmente, hay un amplio consenso: el solo hecho del encarcelamiento del probable responsable de una falta a la norma no resuelve el tema de fondo del problema e incluso se violan los derechos humanos de las personas que son privadas de su libertad (Lippert y Hamilton, 2020). Además, de no lograr la pretendida reinserción, reincorporación o reeducación de los detenidos, que de facto debería ser el objetivo de la separación del sujeto de la sociedad (Caplan, 2006; Freixa, 1978; Zepeda, 2004).

Ante esta situación, los objetivos de la comunidad científica se dirigen a analizar desde diversos enfoques teóricos y epistemológicos las variables que coadyuvan en la generación del problema. La diversidad de las propuestas que han surgido tratando de dar respuesta del por qué, las personas pueden cometer un delito, ha dado lugar a diferentes tipologías y clasificaciones (Alloza, 2001; Vázquez, 2003, entre otros). Entre ellas, destaca la criminologíca, donde se consideran dos categorías generales: a) la criminología designada como tradicional donde se incluyen estudios con fundamento en metodologías denominadas positivistas; en ellas, se encuentran las aportaciones científicas que provienen de las ciencias sociales, de las ciencias jurídicas y de la psicología. b) La segunda categoría se enfoca en el análisis de variables macroestructurales que se orientan al sistema político y económico, la desventaja social, etcetera, entre las que se destaca el posicionamiento teórico de la criminología crítica (Aebi, 2004; DeKeseredy y Dragiewicz, 2018). Por último, mencionar que también hay clasificaciones que observan las unidades de análisis elementales; por ejemplo, las que se enfocan al individuo y la personalidad, los trastornos mentales, como la sociopatía, y las características innatas del individuo; otros propondrán unidades más amplias, donde se reconoce que la persona en estudio es parte de un contexto familiar y social (Farrington, 2002; Samanci, 2010; Torío, Peña y Rodríguez, 2008).

Dentro de este cúmulo de conocimiento previo, se ha reconocido que tanto la construcción del ser humano y la delincuencia no son unidimensionales; más bien se asumen como multidimensionales y multicausales. Es en este sentido, que en el afán de ver un contexto más amplio y no solo intentar estimar un solo evento como variable o factor predictor de la conducta delincuencial, se utiliza una metodología cualitativa, la que nos va a permitir analizar y comprender las autobiografías de una muestra de 
23 internos de un reclusorio en España, los cuales narran diversos acontecimientos vitales estresantes que experimentaron antes de ser detenidos y que afectaron tanto su desarrollo como sus conductas. Los documentos elaborados por las personas privadas de su libertad se estudiaron con la técnica del análisis de narrativas; para su tratamiento se asumió que los registros tenían la forma de un relato. Algunos autores los consideran muy útiles, ya que aportan información relevante sobre la forma en que los individuos interiorizan y dan significado a los acontecimientos vitales (Goodley, 2001; Crossley, 2002).

\section{Aspectos teóricos}

Una de las perspectivas que incluyen un contexto más amplio en el análisis de la salud psicosocial se enfoca en los estresores (Rey-Bruguera, Calonge-Romano y Martínez-Arias, 2017), de ellas, surge también el estudio de los Acontecimientos Vitales Estresantes (AVE) en el desarrollo de la vida de los individuos y su impacto físico, psíquico y social, esta última otorga un marco para estimar diferentes cambios y sucesos, desde el nivel micro hasta el macro social; su importancia radica en la influencia que dichos eventos pueden tener en la identidad, el desarrollo psicosocial y de habilidades de un individuo (Sanz Rodríguez et al., 2009).

Los AVE, son eventos que se han clasificado como parte de los sucesos paranormativos, es decir, aquellos que en mayor medida no los vive la población en su magnitud o frecuencia (Jiménez, Menéndez e Hidalgo, 2008). De acuerdo con Suarez (2010), definir los AVE no es fácil por las diferentes acepciones que se han hecho de ellos; no obstante, él señala que los componentes fundamentales para ser considerados en esta categoría es que son acontecimientos que implican relevancia en la cotidianidad con matices negativos que inducirán una modificación en la vida de los individuos.

$\mathrm{Al}$ hacer una revisión sobre este tema se puede apreciar que se encuentran estudios que describen el impacto de los AVE en la salud psíquica, en el bajo nivel de adaptación psicológica (Lila, Gracia y Murgui, 2013) y en el comportamiento, e incluso otros, de manera indirecta en la integridad física de los individuos.

Entre los estudios que observan los primeros, se encuentran las afectaciones de los AVE por el estrés generado en la vida cotidiana por la edad o la inestabilidad de los individuos, que se origina en los periodos de transición entre la adolescencia y la edad adulta. Zolog et al. (2011) considera la edad como un mediador importante de la ansiedad, siendo los menores 
quienes presentan un mayor nivel; así se constató en una muestra de jóvenes españoles.

Se encuentran también los AVE vinculados a la familia de los individuos estudiados; se argumenta que son los que ejercen un mayor impacto en el bienestar psicológico (Kulkarni y Patwardhan, 2015). Desde la propuesta de Sanz Rodríguez et al. (2009) hay una correlación positiva entre la conducta sintomática y eventos ocurridos en la familia de origen; ellos observaron la presencia de discordancia familiar de alguno de sus miembros, la personalidad externalizante, la manifestación de rebeldía y oposicionismo, entre otros. A su vez, Peleg (2014) ha encontrado que los miembros de una familia que experimentan AVE tienen riesgo de vivir triangulación intergeneracional y una menor diferenciación de sí mismos respecto a otros individuos.

Son consideradas como AVE, por tanto, las relaciones conflictivas y la violencia en la familia - ya sea recibida u observada - (Rey-Bruguera et al., 2017; Sanz Rodríguez et al., 2009). En esta propuesta se encuentran las investigaciones sobre niños, víctimas de maltrato (Kim et al., 2014), que señalan la implicación del maltrato en la infancia y los AVE con el riesgo de desarrollar moderado o severo craving en la edad adulta. De manera similar, Allwood et al. (2012) mencionan que haber estado expuestos a la violencia, de manera temprana, lleva a los jóvenes a perder las ganas de vivir; es el caso de los niños que viven en comunidades pobres y conflictivas; esas situaciones les inducen a la desesperanza; ese estado de ánimo es un mediador de la relación entre los eventos adversos en la vida y la delincuencia.

Ya desde hace más de una década, (Maschi, 2006) indicaba que la acumulación de algunos eventos estresantes vitales aumenta la posibilidad de delincuencia juvenil; se apoya en la teoría que señala que eventos menores generan acumulación en la tensión o la exposición a la violencia, de ahí que deberían ser un factor por investigar. Maschi y Bradley (2008), posteriormente, presentaron hallazgos en relación con la violencia extrema, observaron que los jóvenes que reportaron una participación en delitos violentos, también manifiestan una mayor exposición a la violencia, eventos estresantes, ira y relaciones cercanas con compañeros delincuentes; la ira es la que manifiesta mayor influencia en delincuencia violenta.

La delincuencia toma una dimensión diferente al incluir en el análisis el contexto; desde décadas pasadas se consideraban factores de riesgo: la pobreza (Berti y Pivetti, 2019), vivir en zonas de desempleo, baja supervisión de los padres y los AVE; todos ellos son observados, tanto como fac- 
tor de riesgo, como indicadores para contemplarlos dentro de propuestas de prevención (Hoffmann, 2003). Las razones para considerar el contexto socioeconómico, sobre todo de bajo nivel, se debe a los mayores estresores $\mathrm{y}$ una mayor utilización de estrategias de afrontamiento interno, principalmente al advertir que las familias de nivel medio alto utilizan más el apoyo social como estrategia de afrontamiento (Cracco y Blanco Larrieux, 2015); por último, respecto a esta propuesta, es pertinente señalar que no se puede considerar la pobreza en sí misma como un determinante, sino como parte de los estresores vitales y vinculados a políticas sociales y económicas.

Otra cara del contexto es la violencia, que en la actualidad se encuentra entre las más relevantes, la violencia en las relaciones de noviazgo, que también se ha considerado dentro del análisis de los eventos estresantes en la vida (Chen y Foshee, 2015).

Como se puede observar, las anteriores investigaciones hacen referencia a los AVE y su relación con el bienestar y/o malestar psicosocial, entre los que sobresale la depresión (Fanti, Colins y Andershed, 2019; Jennings et al., 2019), la presencia de diferentes tipos de violencia en el desarrollo de las personas y su relación con problemas posteriores, entre ellos, el que ocupa a este estudio. Es pertinente señalar que, por otra parte, se encuentran otros estudios que no señalan la violencia directamente como AVE, pero sí manifiestan su impacto en el desarrollo de los individuos, como podemos observar en la propuesta del Centro de Investigación para el Desarrollo (CIDAC, 2016) donde se realizó una encuesta a menores infractores detenidos, en ella se corroboró que los entrevistados habían tenido algún tipo de maltrato en sus hogares: insultos, abandono, descuido, humillaciones, castigos exagerados y hasta abuso sexual. A su vez, Mcleer y Dehart (2013) encontraron correlación entre inestabilidad en la adolescencia, desarraigo, movilidad en las escuelas; reportando también la inconsistencia en los cuidadores de los jóvenes como factor importante.

También hay estudios que han sugerido que los AVE se encuentran vinculados al daño físico en las personas (en mayor medida causados por las autolesiones) en los intentos de suicidio. Los suicidios han sido relacionados con la experiencia de violencia y a una menor percepción de apoyo de acuerdo con lo reportado por Guillén et al. (2015). Asimismo, se ha reportado que la letalidad del intento es proporcional a la desesperanza experimentada por las personas que atentan contra su vida (Jaiswal et al., 2016). Grover et al. (2009) registraron que los pensamientos suicidas aumentan con los acontecimientos estresantes o la exposición al estrés en la vida de los estudiantes jóvenes (Sokratous et al., 2013; Phillips, Carroll y 
Der, 2015). Entre los daños físicos vinculados a los AVE, se hallan también los derivados del consumo de sustancias dañinas y las actividades de riesgo. Tamers et al. (2014) señalan el aumento de consumo de alcohol en los individuos durante los acontecimientos estresantes; a la vez que otros estudios relacionan los AVE negativos con población adolescente, indicando que los impactos de ellos fueron predictores de síntomas de ansiedad y de su participación en actividades delincuenciales y otros problemas de conducta (Rubens et al., 2013).

La importancia de observar los problemas de los individuos en el contexto radica en poder ofrecer alternativas para su prevención e intervención desde donde se gesta el estrés, que puede evolucionar en manifestaciones que impactan al individuo y a la sociedad. En la literatura se ha argumentado que los vínculos sociales son importantes para disminuir los eventos estresantes; en primer lugar, se hace referencia a la importancia de las relaciones familiares positivas, tanto para el ajuste interno como externo, de ahí su configuración como factores de protección ante las consecuencias negativas de factores estresantes en el desarrollo de vida de los jóvenes (Oliva et al., 2008). En segundo lugar, para mediar la disminución de la somatización - un aspecto presente en los acontecimientos vitales negativos del individuo - por lo que el mantener vínculos sociales se erige como un medio para el apoyo social (Singh y Dubey, 2015) y el fomento del desarrollo de habilidades para la resolución de problemas y, por ende, como un factor protector en los individuos (Grover et al., 2009). De esta forma, se piensa que mayores habilidades en la resolución de problemas se encuentran asociadas con la menor percepción de eventos negativos en la vida (Eskin et al., 2014). La literatura científica de hoy confirma que la estructura o secuencia de los AVE en la vida cotidiana es parte importante de nuestra adaptación y, por ello, de interés mismo para las personas.

El objetivo del presente trabajo es analizar los relatos de vida de 23 Internos Penitenciarios de una Unidad Terapéutica de España para identificar por medio de sus narraciones cuáles AVE registran en mayor medida en sus historias como parte de su experiencia en la infancia y adolescencia, y evaluar cómo se van entrelazando con efectos psicosociales negativos en su trayecto de vida.

Un aspecto relevante por considerar es que las evaluaciones de los AVE se han realizado mayoritariamente desde la metodología cuantitativa. Sin embargo, la investigación cualitativa en la última década ha tenido una mayor aceptación en diferentes disciplinas (Hoeber y Shaw, 2017). En esta línea, una de las propuestas de la integración de la investigación cualitativa 
a la investigación social señala que con el advenimiento de las nuevas tecnologías las distinciones entre ambas metodologías tenderá a desvanecerse (Leppink, 2017). Se convierte la investigación cualitativa en una alternativa para el análisis de los AVE, en tanto permite incluir la interacción de múltiples variables desde el análisis comprensivo.

Esta metodología tiene como propósito dar sentido o interpretar los problemas sociales en términos del sujeto de investigación (Meo y Navarro, 2009); se parte de la idea de que los relatos dan sentido a las acciones de los individuos y los eventos vividos dan un significado integral al curso de su vida (Cornejo, Mendoza y Rojas, 2008). El análisis de los AVE permite estudiar su impacto tanto en eventos favorables como desfavorables para el desarrollo sano o sintomático de las personas.

\section{MÉTodo}

\section{Participantes}

Estudio con enfoque cualitativo, ex post facto, y de corte transversal, con una muestra de 23 internos varones de un Centro Penitenciario de España, quienes fueron seleccionados por medio de un procedimiento intencionado; estos internos fueron sentenciados por delitos agravados contra las personas y contra el patrimonio. Se consideraron aptos para el estudio a quienes voluntariamente aceptaron participar y estaban cumpliendo una medida judicial en la Unidad Terapéutica del Centro Penitenciario durante el periodo comprendido entre enero y diciembre de 2014. Se tomó una muestra final de 23 internos adultos mediante puntos de saturación durante las fases iniciales de acopio y análisis de los datos. La muestra tiene una amplitud de rango de edad entre los 18 y 68 años (con una media de edad de 34.21 años).

\section{Instrumento y procedimiento}

La observación de la composición y secuencia de los AVE en la trayectoria de vida de los internos, así como su relación, tanto con conductas delictivas como con otros factores moderadores, se realizó por medio de un registro autobiográfico. En el presente estudio, esta técnica permitió hacer inferencias sobre la presencia o ausencia de las variables en estudio, así como de la posible relación entre ellas en puntos críticos de las historias escritas por los internos.

Las historias fueron escritas por los participantes como una condición para acortar su sentencia acudiendo a una Unidad Terapéutica. Para in- 
gresar a ella, seguir un proceso de terapia y de pase del estado de queja al reconocimiento del delito, los sentenciados tenían que ofrecer un relato autobiográfico sobre sucesos vitales, experiencias y sentimientos personales hasta el momento en que cometieron el delito por el cual fueron recluidos. Los participantes recibieron información oportuna respecto a las dimensiones, límites y fines del procedimiento, con el objetivo de que su participación estuviera ajustada a un claro conocimiento de su función como sujetos de investigación. Posteriormente, se obtuvo su consentimiento informado, debiéndose resaltar que se contó para ello con el permiso de las autoridades administrativas, así como también de las comisiones técnicas y éticas implicadas en la Unidad Terapéutica.

La redacción de las historias fue controlada por medio de consistencia y contraste con el registro de la historia penitenciaria. Conforme con este criterio, el delito cometido por los internos fue identificado en la base de datos del Centro Penitenciario. Posteriormente se construyó una matriz para establecer sistemáticamente la relación del delito con los AVE mencionados en los relatos; ello bajo el supuesto de que el relato autobiográfico haría referencia a AVE previos a la comisión del delito por el cual fueron sentenciados.

Los internos escribieron en papel sus informes autobiográficos, los que fueron transcritos posteriormente en el procesador de textos Word para su análisis con el programa de cómputo MaxQda 18. De acuerdo con algunos requisitos metodológicos, se excluyeron del estudio los casos en que se detectó, a través de un análisis de texto, que los informes no ofrecían un contenido ajustado al formato del informe autobiográfico solicitado por las autoridades de la Unidad Terapéutica. Los análisis de relatos con este programa arrojaron información confiable a partir de la concurrencia de indicadores entre casos y de su presencia consistente en cada uno de ellos.

\section{Técnica de análisis}

Los informes autobiográficos fueron estudiados con la técnica del análisis de narrativas; para su tratamiento se asumió que los registros tenían la forma de relatos. Goodley (2001) y Crossley (2002) los consideran muy útiles, ya que aportan información relevante sobre la forma en que los individuos interiorizan y dan significado a los acontecimientos vitales. De esta manera es posible indagar la manera en que los sujetos conectan diversos acontecimientos a través de sus experiencias vividas; en congruencia con esta perspectiva, Josselson (2006) afirma que el análisis de narrativas se concentra en los significados que las personas usan para ordenar y conectar 
sus vivencias. Esto nos lleva a enmarcar el estudio realizado en el análisis de los informes autobiográficos de los sentenciados para identificar la forma en que perciben los AVE y su relación con estados afectivos previos a la comisión de delitos.

El presente estudio se ha basado en la técnica conocida como análisis paradigmático de contenidos, a través del cual se exploran las similitudes y diferencias en distintas historias. La aportación de esta técnica radica en extraer generalizaciones sobre los temas centrales que componen los relatos de los individuos.

El análisis paradigmático, siguiendo a Lieblich, Tuval-Mashiach y Zilber (1998), consiste en fragmentar el texto y someter cada parte de un relato a un examen de contenidos y significados. Dependiendo del objetivo del estudio y del material narrativo, existen muchas variantes de este tipo de análisis. La utilización de una u otra depende del criterio que tienda a destacar en el procedimiento. Por un lado, puede sobresalir la saturación cuantitativa de temas contenidos en los relatos; por el otro, puede ser saliente el tratamiento hermenéutico de los significados que los sujetos dan a los acontecimientos mencionados en sus relatos. No obstante, ambos procedimientos se pueden complementar en ciclos sucesivos. En este estudio se siguió la combinación de ambas técnicas del análisis paradigmático; en concreto, se llevó a cabo en las siguientes fases:

1. Inicialmente se aplicó un análisis textual al informe autobiográfico con el fin de detectar inconsistencias en la estructura de los relatos y verificar la honestidad de los mismos. De ellos, 23 fueron sometidos al análisis paradigmático de narrativas con el fin de detectar posibles asociaciones entre las variables en estudio.

2. Posteriormente, se desarrolló una codificación abierta, axial y selectiva para categorizar los AVE, los tipos de delito y la posible presencia de factores moderadores de los AVE, de manera que se descubrieran también posibles relaciones entre categorías de dichas variables.

3. Luego se realizó un análisis comparativo de las dimensiones y categorías comunes de AVE, tipos de delitos y la posible ausencia de factores moderadores comunes en algunos relatos.

4. Se clasificaron los casos a partir de las asociaciones observadas entre la composición de categorías predominantes de los AVE y de los estados afectivos, previos a la comisión de delitos.

5. Se obtuvieron los significados de los temas principales de los relatos.

6. Se analizó la consistencia de los temas intra e inter casos. 
7. Se obtuvo, por inducción, las relaciones entre las categorías de AVE y otras variables en los relatos analizados.

\section{Resultados}

Asumiendo la presencia de AVE en la vida de las personas como parte importante de su adaptación, se considera de interés la estructura o secuencia de los mismos en las personas, para lo cual se realiza el proceso de lectura y relectura de los relatos de vida por medio de la codificación para obtener códigos y categorías de los eventos vitales estresantes y, posteriormente, realizar un análisis comparativo de los códigos comunes encontrados en los relatos; ello posibilitó proceder a la ordenación conceptual de grandes categorías para confirmar el marco teórico inicial de los AVE presentes en los individuos entrevistados.

El análisis de la coocurrencia de los códigos en esos escritos nos permitió descartar aquellos que no coinciden de manera frecuente en las historias $y$, a la vez, identificar los que convergen con más frecuencia. Por ello, se descartaron otros AVE presentes como las penurias económicas, la muerte o enfermedad de algún familiar directo, cambios de residencia, entre otros; se destacan como generadores de estrés en este estudio: estilo educativo, con 45 segmentos presentes en las 23 historias analizadas; eventos en la trayectoria escolar, con 40 segmentos obtenidos en 19 de los 23 relatos de vida: contacto temprano con drogas, con 27 segmentos en 19 de los relatos: y la presencia de familiares adictos cercanos al entrevistado, con 22 segmentos de un poco más de la mitad de las entrevistas, a la vez que violencia en el interior de las familias (ver Figura 1).

Estos eventos presentes en la vida de los internos penitenciarios, de acuerdo con la teoría de los AVE, se catalogan como acontecimientos paranormativos, es decir, que no son esperados o deseables para el desarrollo competente de los sujetos (Jiménez, Menéndez e Hidalgo, 2008). Estos eventos paranormativos o vitales estresantes en este grupo de personas, por su frecuencia, pueden clasificarse en tres categorías formadas cada una por tres componentes (ver Figura 2).

1. Acontecimientos en inicio del desarrollo sintomático: Estilo educativo/relacional y Trayectoria escolar.

2. Acontecimientos estresantes que mantienen la intensificación de síntomas: Adicciones y delito.

3. Acontecimientos derivados de las relaciones íntimas: Violencia intrafamiliar y Ruptura de pareja. 
Con los anteriores datos, se inició la construcción de un mapa de los AVE de acuerdo con su aparición en las narraciones de los participantes.

Figura 1: Categorías encontradas de los AVE en los relatos de vida y número de documentos

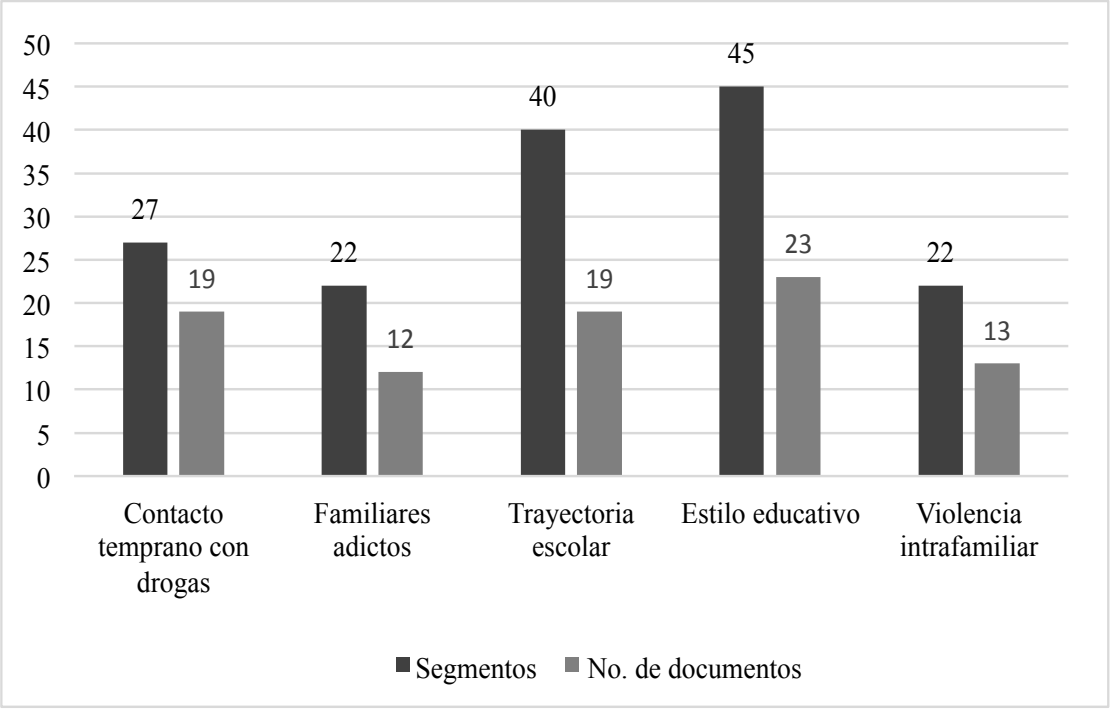

Fuente: elaboración propia.

\section{Acontecimientos en el inicio del desarrollo sintomático: Estilo Educativo/relacional y Trayectoria escolar}

Comenzando con el código con más presencia en los relatos de vida y con una manifestación temprana en la vida de los entrevistados; esto es, la referencia a eventos estresantes en el inicio y desarrollo educativo en el sistema formal de educación y en el estilo de educación y, vinculado a ello, el estilo en las relaciones que se establecen en el sistema parental en ese proceso; los resultados refieren: en primer lugar, el estilo "dejar hacer" (52.2 por ciento) en la forma de relacionarse las figuras parentales con los hijos; le sigue el "estilo autoritario" (30.4 por ciento), destaca aquí que no se encontraron narraciones de un estilo autoritativo, como la teoría lo refiere (Baumrind, 2005); pero sí un tercer estilo, que transita entre el "ser autoritario y el dejar hacer" (17.4 por ciento). 


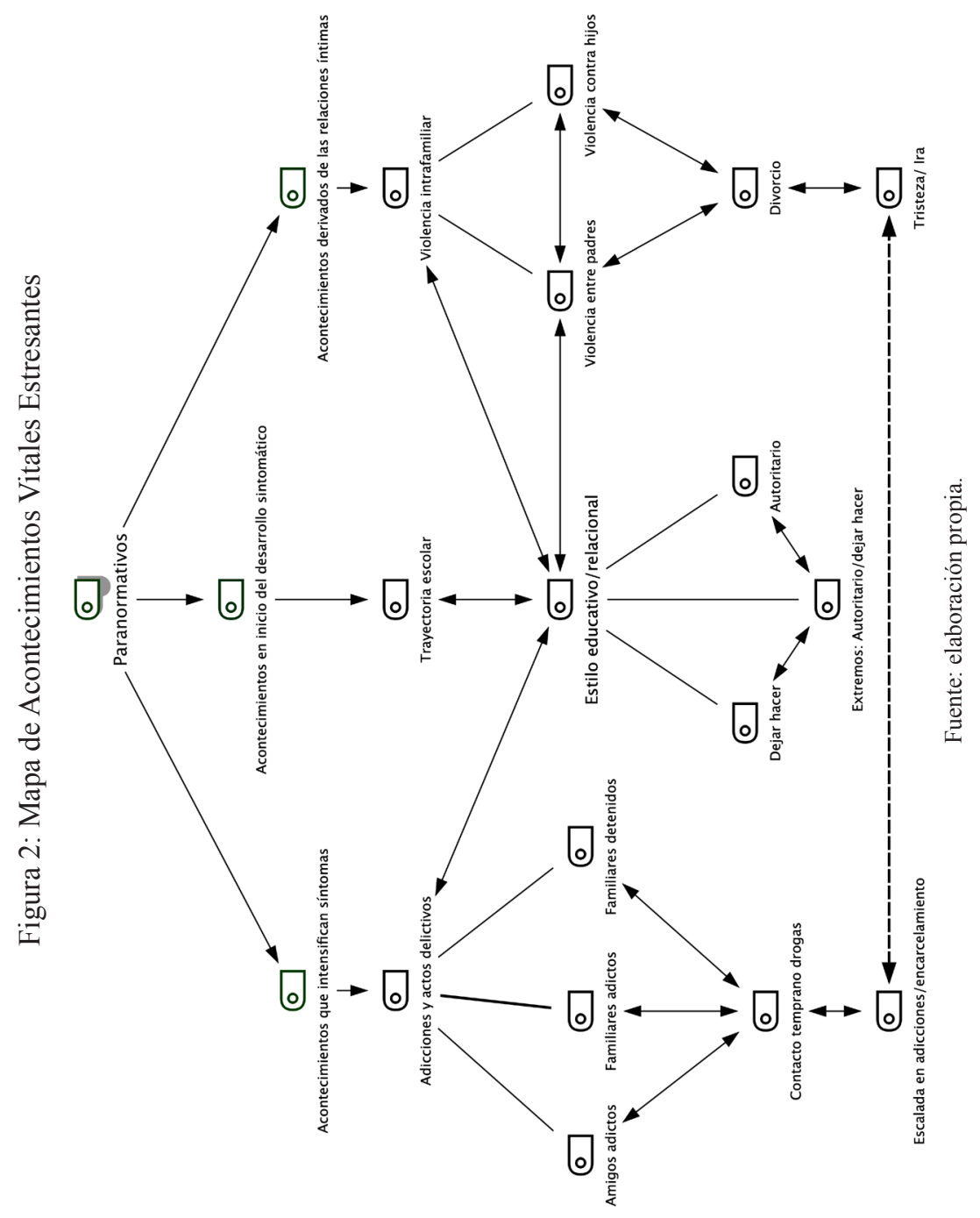


Dejar hacer. Este código se encontró en las narraciones de más de la mitad de los participantes; se refiere a los significados atribuidos por los internos al cuidado familiar, que señalan el nulo, o escaso control sobre horarios de llegada y salidas del hogar, así como falta de atención hacia las directivas, normas y acciones dirigidas a los hijos pequeños. Lo ejemplifican algunos extractos en las narraciones a continuación:

(...) Yo de niño ya estaba robando coches y comercios, con gente que era mayor que yo, en casa no tenía horario, llegaba, a las tres o cuatro de la mañana o no iba a dormir y no pasaba nada; hasta que un día robé una bez, [sic] un 124 sport... (4 $\left.\mathrm{CPE}^{1}\right)$.

Mis padres no se enteraban porque trabajavan [sic] mucho, para que no nos faltase de nada. La única que me controlaba un poco hera [sic] mi hermana, pero cuando yo tenía 14 años se casó. Así es que ya tenía el camino libre para hacer lo que quisiese... Me hecharon [sic] varias veces de el [sic] instituto, pero yo me las arreglaba, para que mis padres no se enteraran. Los vecinos empezaron ha [sic] hablar y a decirles a mis padres, que yo no andaba bien, por supuesto mis padres me creyeros a mí, cuando yo les dije que eso no hera [sic] verdad (6 CPE).

...Tengo que decir que mi padre nunca me impuso respeto, nunca me reñía y siempre me explicaba las cosas tranquilamente, mi madre me explicaba a través de la riña, nunca me llegaron claras las explicaciones que mis padres me daban a la hora de corregirme ya que no les hacia $[s i c]$ ningún caso y siempre hice lo que me dio la gana (21 CPA).

Autoritario. Este estilo se encontró presente en 30.4 por ciento de las autobiografías. Las narraciones de los participantes hacen referencia a la percepción de la autoridad rígida de parte de alguno de los padres como medida para corregir la conducta de los hijos, y a la resultante agresión sufrida en la infancia:

(...) Me pegaba con los compañeros, contestaba, en fin era un cafre, me empezaba en casa a portar mal, me escapaba cuando me castigaban y en sexto me dieron mi primer porro y me gustó; en el cole ya no me querían me expulsaban. Y cuando llegaba a casa mi madre me pegaba me castigaba pero yo solo le hacia [sic] caso a mi hermano (23 CPA).

Era tímido y muy mal estudiante. Me juntaba con los pocos amigos que tenia [sic] a hacer gamberradas de mal gusto... la más fuerte que recuerdo que [sic] tirar una piedra a la autopista y provocar un accidente... Al llegar a casa mi padre me propino [sic] una paliza brutal con puñetazos, patadas, tirones de pelo y demas $[s i c] \ldots$ (15 CPA).

${ }^{1}$ Indica los números otorgados a cada autobiografía que integra la muestra y el tipo de delito del que se les acusó. Delitos contra las personas (CPE), delitos contra el patrimonio (CPA). 
(...) Eramos $[\mathrm{sic}]$ siete hermanos, nunca nos faltó de comer, mi padre, exepto [sic], cuando bebía, que me pegaba, cratuitamente [sic], fue pasando el tiempo, cuando no iba a la escuela, que eran las mayorías de las veces, ya que solia [sic] pirarla mucho (8 CPE).

Autoritario/dejar hacer. Este tipo de relación se encontró en los significados contenidos en 17.2 por ciento de las autobiografías. Lo que caracteriza esta manera de ejercer la autoridad de las figuras parentales es, por una parte, el ejercicio de la violencia a través de los golpes para tratar de imponer su autoridad o sus directivas, pero con falta de ejercicio efectivo de las indicaciones dadas a los hijos; por otra parte, se observa que la forma de ejercer la autoridad de los encargados del cuidado de los niños es llevar a cabo acciones de manera polarizada o no sincronizada.

(...) Mi padre recuerdo que nos reñía por estar siempre por la calle jugando y no respetar los horarios, también nos solía pegar cuando se enteraba que robamos en los bares, aunque yo no le hacia [sic] mucho caso. Me decía que le dolia $[\mathrm{sic}]$ a el mas $[\mathrm{sic}]$ que a mi [sic] cuando me pegaba y alos [sic] pocos días estaba en la misma. Mi padre me decía que la prosima [ $\mathrm{sic}$ ] vez que le digan algo que no iba a salir de casa. Yo con esta edad desde los ocho años piraba clase, engañaba a mi abuela mintiéndole que iva [sic] a clase y me quedaba jugando con mi hermano y mis amigos (10 CPE).

La relación con mis abuelos era bastante buena, pero es verdad que era bastante respeto, y en especial a mi abuelo porque como ya les dije antes, le gusta mucho la disciplina. Con mi abuela era diferente, me mimaba mas (ileigble) y a veces me compraba golosinas a escondidas de mi abuelo, porque él decía que comer esa mierda te picaba los dientes (19 CPA).

Recuerdo tambien [sic] que mi madre nos encerraba por qué [sic] no podia [sic] con nosotros. No nos podia [sic] ni bañar por qué [sic] mi hermana y yo nos escapabamos y nos ibamos [sic] cada uno por un lado, así que no nos en cerraba $[$ sic $]$ y ya está (11 CPE).

Vinculado con el código anterior, encontramos los significados que tiene el desarrollo vital en periodo escolar o, como hemos denominado, trayectoria escolar. Destaca que 72 por ciento de los participantes ha manifestado algún evento estresante en este periodo: internamientos en colegios por la separación de los padres, cambios de un centro escolar a otro, fugas del colegio y peleas con compañeros, entre otros.

(...) Con esa edad nos ingresan en un colegio internos... y eso nos hace sentir miedo y respeto por lo que nos ibamos [sic] a encontrar, tenia [sic] miedo a ser rechazado por la gente por ser gitano y que nos pegaran y trataran mal, fue 
entonces donde durante un año de estancia aprendimos a leer y a escribir, de vez en cuando nos visitaban nuestros padres ... Un dia [sic], aprovechando el descuido, nos escapamos y nos plantamos en casa de nuestros padres, estos por miedo a que nos regresaran otra vez al colegio, deciden que nos vallamos [sic] de la ciudad... (2 CPE).

Durante el trascurso de mi vida me cambiaron varias veces de colegio la primera fue cuando nos echaron de esa vivienda que estábamos de ocupas [sic] porque no quería ir a ese colegio que estaba al lado de donde estábamos en la calle y cuando salía al recreo se reían de mi [sic] los niños y mis padre decidieron cambiarme de colegio... Estuve así hasta los 16 años siendo un bala perdida sin importarme nada asta [sic] que por mi edad me echaron del colegio sin acabar los estudios básicos... (7 CPE).

Como iba diciendo, yo seguia [sic] pirando clase, asi [sic] que un dia [sic] que fue mi hermana la mayor mandada por mi padre para pagar el mes, de clase, la maestra le dijo, a mi hermana, Qué [sic] no me cobraba puesto que no seria [sic] ético por su parte ya que tu hermano (se refiere a él mismo) vendria [sic] 15 dias a clase, y estais [sic] tirando el dinero, sin que os sirva para nada, la buena señora obra como pocas personas obran en el transcurso de mi vida (8 CPE).

A los ocho años... me apuntan al colegio a mi [sic] no me gustaba ir. Recuerdo que me peleaba con los niños porque no queria [sic] estudiar y luego la profesora me castigaba. Le cogi $[s i c]$ mucha rabia porque aunque no tuviera la culpa me castigaba igual siempre a mi [sic] (9 CPE).

En el colegio me solia [sic] pegar con mis compañeros, no me gustaba perder y me pegaba con mis amigos. Solía pirar $[\mathrm{sic}]$ clase con mi hermano, me ponía de acuerdo con mi hermano para robarle dinero a mi abuela pa [sic] gastarlo mientras pirábamos clases me costaba mucho estudiar (10 CPE).

Las anteriores descripciones permiten contrastar la percepción del estilo educativo o relacional ejercido por las figuras parentales; consideramos que el estilo educativo es también un estilo relacional; es decir, una forma de organizarse y relacionarse con los hijos por parte de las figuras paternas. Se observa en las narraciones que no se aprecia una forma consistente, una guía para definir las acciones de los hijos; mucho menos se observa el estilo autoritativo que la literatura señala como el que apoyaría un desarrollo sano de los hijos, por ello, es congruente el hecho de que no aparezca este estilo en ningún relato de algún integrante de la muestra. Igualmente, en las narraciones no se observa un seguimiento de las actividades escolares de los hijos, donde se manifiesta de manera recurrente el faltar a clase, peleas; pareciera que los padres ni se enteran, ni participan en la formación de los hijos de acuerdo con el discurso de los participantes. 


\section{Acontecimientos estresantes que mantienen la intensificación de síntomas: adicciones y delito}

Las diversas autobiografías, en su secuencia narrativa relacionada con su inserción en el sistema educativo, señalan que las figuras parentales parecerían no seguir un objetivo en cuanto al desarrollo de los menores de edad, incluyen en este periodo convulso de su vida el contacto con personas cercanas que usan drogas (68 por ciento) - entre ellos, familiares de los participantes (48 por ciento) - y, consecuentemente, el inicio del consumo de sustancias adictivas y el desarrollo de las mismas en la niñez y adolescencia en un importante grupo de participantes.

(...) Yo cuando me escapaba me dedicaba a pedir o robar por las tiendas para poder comer y a veces me ponía a buscarme la vida con una guitarra y asi [sic] sacaba dinero para comer y para comprar porros ya que comence [sic] sobre la edad de ocho años a fumar y a esnifar pegamento ya que donde vivia [sic] en el poblado habia [sic] muchos chavales que lo hacian [sic]y yo para no ser menos lo hacia $[s i c]$ tambien $[s i c]$, si soy sincero cuando fumaba y esnifaba pegamento me olvidaba de todo (2 CPE).

...Adoraba a mi padre y todo su mundo hera [sic] de alguna manera mi hidolo [sic], me enseño [sic] a conducir, a montar a caballo, las armas y todo su mundo incluido las drogas. Con la edad de trese [sic] años empece [sic] a fumar porros. Y mi padre se entero [sic] y mi padre ya lo sabia [sic] me llamo $[s i c]$ aparte y me pregunto [sic] si es que yo fumaba yo le dije la verda [sic]. $\mathrm{Y}$ aunque tenia $[s i c]$ miedo por lo que me pudiera hacer, lo unico $[s i c]$ que me hizo fue hacerme coger tal colocon [sic] que me puse malisimo [sic]. El [sic] lo hizo para ver si le cogia [sic] miedo o asco, pero no lo consigio [sic] (3 CPE). Mis primeros contactos con la droga yo tenia [sic] 12 años y empezaba [sic] observar que mi hermano fumaba porros yo empeze [sic] a fumar tabaco solo a escondidas de mi hermano porque me comparaba con el [sic] y me sentía inferior a el [ $[s i c]$. Sentía envidia y así empese [sic] a fumar dejándome llevar por sus amigos a escondidas de el [sic], yo tenia [sic] miedo de que se enteran [sic] que yo fumaba tabaco y cuando me preguntaban les mentia [sic] por miedo de que se enteren que yo fumaba (10 CPE).

...En el colegio fue $[s i c]$ mal me fugaba a los diez años empecé a juntarme con chavales mayores que yo con solo once años ya fumaba con doce fumaba porros ya con esa edad robaba tonterías camisas y demás con trece ya tiraba rallas $[s i c]$ hasta los quince años que ya estando en el barrio subí a casa de un amigo y saco $[$ sic $]$ una platina y echo $[$ sic $]$ caballo (27 CPA).

El desarrollo vital descrito por una falta de directivas, escasa o nula observación del desempeño escolar de los hijos, e incluso, normalización 
del uso de sustancias legales e ilegales en la familia, no puede extrañar que favorezca el consumo de sustancias, de manera temprana, de los participantes. Así lo confirman las narraciones sobre el uso y posterior abuso de ellas, y su implicación en actos delictivos para dar paso a un mayor consumo, hasta su detención; ello explica, pues, un sector importante en el desarrollo temprano de nuestra población participante: la categoría a denominar como Violencia intrafamiliar y Ruptura de pareja.

\section{Acontecimientos derivados de las relaciones íntimas: violencia intrafamiliar y ruptura de pareja}

En las narraciones que describen la manifestación de violencia intrafamiliar, la más recurrente en los discursos es la vivida entre los padres; en su mayoría, las narraciones coinciden en la dirigida a la madre por parte de su pareja: 52 por ciento presencia o vive violencia. Se constata, así, que los entrevistados viven y sufren una relación violenta del padre hacia la madre, generando miedo y sentimientos de impotencia en los hijos por las manifestaciones de violencia en los padres, donde también se da la misma frecuencia de padres divorciados (52 por ciento).

(...) Recuerdo también que cuando llegava [sic] mi padre del trabajo sino [sic] le gustaba la comida discutia [sic] con mi madre, mi hermana y yo nos sentíamos muy mal ya que teníamos miedo y eramos [sic] muy pequeños. Gritaba mucho y se escuchaba en todas chovolas [sic] (9 CPE).

...Comienza cuando tenía nueve años pues mi padre empezo [sic] a beber alcohol y a maltratar a mi madre lo cual yo desde mi habitación lo oía todo y me sentia [sic] muy triste y a la vez muy impotente y con mucha rabia... (18 CPA). Con ocho años mi madre y mi padre se separan porque mi padre era alcoholico $[s i c]$ solian $[s i c]$ discutir mucho. Me generaba mucha ravia $[s i c]$ malestar. Mi madre nos dejo $[s i c]$ en casa de mi abuela debido a la situación (10 CPE).

La violencia narrada no solo es la dirigida por el padre hacia la madre; se encuentra cristalizada igualmente en relaciones de figuras parentales hacia el entrevistado, en otros integrantes del sistema fraterno, o de los hijos a los padres.

(...) De pequeño mi padre me apunto [sic] en la escuela... pero no me dejo [sic] ir a pesar de que yo quería ir, a mis hermanas, que si [sic] que las dejo [sic] ir a la escuela pero a mi [sic], esto era algo que yo no entendía y me hacia [sic] sentir mal ....yo no podia [sic] porque mi padre no queria [sic]y yo tenia [sic] ganas de ir pero no lo hacia [sic] por miedo a que mi padre me pegara y me castigara. Yo recuerdo que mientras los demás se ivan [sic] a la escuela yo me 
quedaba en casa solo jugando con la tierra, el perro y los gatos que eran mi única compañía, me sentía muy solo y sin nadie a mi lado que me diera afecto y cariño, solo tenía a mi perro. Mi padre siempre que llegaba a casa le pegaba palizas al perro y yo me ponía a llorar porque me daba mucha pena porque lo queria $[\mathrm{sic}]$ mucho, yo me quedaba muy mal, me daba mucha rabia y la cogía dándole patadas a la furgoneta de mi padre y el [sic], cuando me veía, me pegaba a mi $[$ sic $]$ (13 CPE).

Las cosas en casa iban bien hasta que el padre de mi hermana le empezó a pegar a mi madre por culpa del alcohol y a mi [sic] eso de ver como [sic] le pegaba a mi madre me rompía el alma en dos ya que a mi madre yo siempre la quise con locura y yo no la podía ver sufrir. Yo lloraba mucho por mi madre y me sentía muy impotente por que [sic] no podía hacer nada para evitarlo y a mi [sic] me daba mucha pena ver como lloraba y sangraba por todos los sitios yo en esa época tenía diez años (20 CPE).

Mi padre me (ilegible) dio una paliza brutal con puñetazos, patadas, jalones de pelo y demás, yo buscaba cariño en mi tío José, no era la primera vez que me pegaba pero nunca con tanta violencia, mi padre era una persona con problemas con el alcohol, yo en casa veía esta actitud de mi padre hacia mi madre, lo que me hacia [sic] sentir odio, rechazo y miedo hacia [sic] él. Yo me ponía a llorar atrás de la puerta de su habitación, mientras hacia $[s i c]$ insultos y chillidos (15 CPE).

La relación familiar no era muy buena dado a que con catorce años me echaron de casa por que $[$ sic $]$ me pege $[$ sic $]$ con mi padre tras ver como discutía con mi madre después de lo que marchaba my $[\mathrm{sic}]$ padre me dio con algo en la cabeza y me la abrió de eso nunca me olvidare [sic] (23 CPE).

En algunas historias parecería que la violencia es el desencadenante de la separación. Las madres cansadas de los malos tratos optan por buscar una vida diferente; esas descripciones no pueden ser corroboradas y debemos entender que son las construcciones significativas que ellos han elaborado a través del tiempo. La versión ofrecida por nuestros sujetos puede verse influida por diferentes variables en su infancia. Es relevante que ellos perciban el sufrimiento de la madre por golpes o gritos que recibe, pero no quieren que siga pasando, y no tienen la forma de evitarlo; la violencia al igual que la separación de sus padres es un hecho doloroso, y en la edad adulta lo siguen narrando con aflicción.

\section{Discusión}

Los AVE son sucesos inesperados e inusuales que modifican de manera significativa las relaciones, rutinas y el contexto de la vida de los individuos; su recurrencia interfiere en el desarrollo de los sujetos, generando 
estrés e incertidumbre en la vida, pero sobre todo si aparecen en edades tempranas. Hasta el momento existen muchos estudios que han medido su presencia, su impacto y su correlación en diferentes problemas psicosociales, entre los que encontramos la violencia en novios (Chen y Foshee, 2015), consumo de sustancias (Tamers et al., 2014), en la manifestación de la delincuencia (Maschi, 2006), y en el del bienestar (Kulkarni y Patwardhan, 2015), entre otros.

Todos los estudios anteriores sustentan la correlación de los AVE con la manifestación de uno u otro problema en la vida de los individuos. Este estudio pretende no solo identificar los AVE en un grupo de personas que han tenido conflicto con la ley, sino también aprovechar las herramientas de la metodología cualitativa para analizar las autobiografías de los internos en el medio penitenciario e identificar, por medio de sus narraciones, los AVE que han experimentado en su infancia y adolescencia, y la forma en que estos se entrelazan en su trayecto de vida.

Las herramientas del software de análisis cualitativo nos permitieron elaborar una configuración del desarrollo de diversos acontecimientos, los que se han clasificado en categorías de acuerdo con su contenido y estructura. Una vez elaboradas las categorías, a través de un análisis de coocurrencia de códigos, se obtuvieron las categorías con mayor presencia en la historia de vida.

Las narraciones de los AVE en el Inicio del Desarrollo Sintomático, donde se hace referencia al estilo Educativo/relacional y a la Trayectoria escolar han ocupado un lugar predominante en cada uno del total de los documentos, al ser descrita la trayectoria escolar problemática en 19 de 23 documentos; en ellos se han encontrado 40 segmentos donde se relata la experiencia. Tomando en cuenta el peso que la categoría del estilo relacional y el desarrollo escolar tiene en las autobiografías, y por ser los primeros acontecimientos de la vida de los participantes, nos lleva a iniciar sobre ellos el desarrollo de la configuración del mapa. En concordancia con los resultados de este estudio, en la literatura se encuentran evidencias del impacto de los AVE en la infancia: aceleran diversos síntomas y más baja adaptación en los niños (Rey-Bruguera et al., 2017), vinculados a las relaciones familiares y a la educación formal; a ellos se les ha atribuido una influencia significativa en los estudios de conductas disruptivas. Ya a inicios del siglo, Hoffmann (2003) consideraba el impacto de baja supervisión de los padres en el desarrollo de conductas delictivas. Posteriormente, Allwood et al. (2012) sostiene que la falta de orientación de los padres debe considerarse en el estudio de la delincuencia. 
En esta línea, las narraciones de este grupo de personas, al describir su experiencia, señalan que la forma de relacionarse de sus padres con ellos, en etapas tempranas de la vida, lo hacen preferentemente desde dos de las tres posibilidades del modelo propuesto por Baumrind (2005): el dejar hacer y la relación educativa autoritaria; en las primeras se describen como niños autónomos, sin la supervisión que Allwood et al. (2012) y Hoffmann (2003) mencionan como una variable que incidirá en el posterior desarrollo de actividades delincuenciales. En el dejar hacer se narran los participantes como infantes que no asisten de manera regular al colegio, llegan tarde a casa, se vinculan con consumidores de sustancias dañinas o realizan alguna actividad ilícita y, en ese discurso, no hay indicios de que los padres se enteren o realicen acción alguna para evitarlo. Por otra parte, hay narraciones que hacen referencia a figuras parentales que ejercen su función de manera autoritaria, en esos discursos manifiestan su malestar por los actos violentos implementados por los padres como una herramienta para tratar de controlarlos; sobre este tema se ha reportado en algunas investigaciones que la violencia no ayuda al control y puede ser un predictor de otros síntomas en los jóvenes (Maschi, 2006; Kim et al., 2014; Guillén et al., 2015); esta situación, a la vez, se encuentra vinculada con la no aceptación del participante de la autoridad paterna y materna y con la infracción de la norma a pesar de los golpes. Ese ejercicio poco efectivo de la autoridad es notorio también en las narraciones donde se describe una combinación entre la pasividad del dejar hacer y la severidad de los intentos de corrección ejercida por medio de golpes por los integrantes del sistema parental, entre los que se encuentran integrantes de la familia extensa - abuelos y tíos, entre otros- Este estilo parental que trascurre en los extremos de la autoridad blanda y dura se observó igualmente en relatos de vida en un grupo de personas internas en un centro de detención en México (Estrada-Pineda et al., 2015). Los resultados de Sanz Rodríguez et al. (2009) constatan también la presencia de desacuerdos entre figuras parentales en la forma de llevar la disciplina, donde uno de los padres es autoritario y el otro se ubica en el extremo contrario, y la posterior manifestación de rebeldía u oposición de algún miembro de la familia.

Se aprecia que los discursos de referencia a la educación formal describen también situaciones de dinámica familiar. La trayectoria escolar queda marcada por distintos AVE, entre los que se encuentran: cambio constante de centro en el que se estudia, el internamiento en colegios y el mal rendimiento escolar con fugas e inasistencias - donde se hace palpable la pérdida de control de las acciones de los infantes-, la tristeza, el miedo 
y la dificultad que los jóvenes viven en su proceso educativo ante la inestabilidad de los dos sistemas en los que interactúan a esa edad; es notable que hasta ese momento no se encuentre en los relatos una intervención de otros sistemas formales de apoyo o su inserción en programas preventivos para atender los diferentes problemas ya evidentes en el desarrollo de los menores.

Además, en esta etapa de la vida escolar donde los jóvenes ya manifiestan problemas, como un componente adicional se encuentra su vinculación con otros jóvenes con problemas similares y consumo de alguna sustancia dañina, legal o ilegal, como tabaco, alcohol y marihuana. Con estas relaciones buscan filiación y pertenencia, así como sentirse fuertes y estar en el nivel de riesgo en que los otros jóvenes están. Pero hay casos donde esa necesidad de pertenecer a un mismo grupo a través del consumo de las mismas sustancias viene del propio sistema familiar, de sus hermanos o del mismo padre; los participantes narran ver el consumo de sus parientes y querer ser iguales que ellos. Todas estas situaciones generan sentimientos de desamparo y estrés en los chicos.

El aspecto concomitante con el uso de sustancias son los actos delictivos. Se ha señalado que el estrés es un factor para el aumento del consumo de alcohol (Tamers et al., 2014) e, incluso, el estrés se traduce en ansiedad, la que a su vez está vinculada con su participación en actos delictivos (Rubens et al., 2013). De igual forma, las narraciones del grupo mencionan que el consumo les otorga filiación, y los actos delictivos se desarrollan de manera paralela a esa dinámica. Se vislumbra la relación entre robar para consumir o consumir para robar, y calmar la tristeza e ira que les producen otros acontecimientos vitales, entre ellos, la violencia intrafamiliar.

Como parte de la espiral de la secuencia de los AVE y el mal funcionamiento escolar, el involucramiento con pares adictos, desarrollo de adicciones e inicio en actividades delictivas, se añaden los conflictos de las relaciones entre los padres; en las autobiografías se describe un mal funcionamiento conyugal: se manifiesta violencia en la relación de pareja (en mayor medida dirigida a la madre), violencia bidireccional, de los padres a alguno de los hijos (hostilidad, intentos de agresión física, gritos, etc.), y de la persona participante en este estudio hacia sus padres. Entre nuestros integrantes de la muestra, ello genera sentimientos de impotencia, desvalimiento, miedo, entre otros. Algunas de las historias exponen la separación de los padres como consecuencia de la violencia, y una gran tristeza por tener a sus padres separados; la tristeza nuevamente remite al participante al consumo de sustancias; en esa secuencia de acontecimientos, resulta re- 
levante también la carencia de narraciones donde se manifieste la presencia de fuentes de apoyo social, ya sea formal o informal que den otro curso a la vida de los individuos por su importancia en el impacto ante los AVE, como se ha planteado en algunos estudios (Lila et al., 2013).

Se puede observar en las narraciones de los integrantes de la muestra que hay un grupo de AVE comunes entre ellos que se entretejen secuencialmente en la experiencia de vida, esto no quiere decir que los participantes no han vivido otros acontecimientos que hayan impactado sus emociones, como los duelos, las enfermedades y los desalojos, por mencionar algunos; estos también se encontraron en menor proporción en las autobiografías de la muestra. Es, precisamente, la frecuencia y la coocurrencia de eventos lo que ha podido configurar un mapa de la forma como se estructuran y se vinculan entre sí. No podemos decir dónde inicia esta espiral de AVE. Es probable que los padres de los internos ya hayan vivido sus propias adversidades derivadas del propio contexto donde han participado; tampoco en este estudio se hizo un acercamiento directo a los estilos de afrontamiento ni a las habilidades de resolución de problemas (Eskin et al., 2014) de las figuras parentales y ni del resto de los integrantes de la familia, ambos puntos son líneas por desarrollar en futuras investigaciones. Lo que resulta evidente en este grupo de personas es que hay una acumulación de eventos estresantes como ha sido constatado en otras investigaciones: en los primeros años de su vida escolar (Herrero et al., 2016), una escasa respuesta o habilidades de los padres para enfrentar eventos estresantes (Grover et al., 2009), y un insuficiente apoyo social tanto formal como informal, debido a que las personas en las que se apoyan los internos son sus compañeros de consumo de sustancias dañinas y de actividades delictivas, por lo que no cumplen con el rol de recurso favorable en casos de AVE como lo han considerado algunas propuestas (Singh y Dubey, 2015). Por lo tanto, ante la turbulencia de acontecimientos estresantes, solo sobreviven sin tener la posibilidad de poder generar recursos para enfrentarlos y superarlos. Por último, señalar que no se pretende hacer generalizaciones, dada la naturaleza del tipo de investigación que sustentamos en este trabajo, sino ampliar el foco de la situación-problema y realizar un acercamiento a la comprensión del proceso de este fenómeno social para que los profesionales tengan un referente para el diseño de estrategias de intervención, así como para la propuesta de políticas sociales dirigidas a la prevención. 


\section{LIMITACIONES Y ORIENTACIONES A FUTUROS ESTUDIOS}

El presente estudio tiene algunas limitaciones que es necesario destacar. Para realizar este trabajo se tomaron como base solamente las autobiografías de las personas que decidieron colaborar en él, pero no se tuvo contacto más directo con los participantes para obtener información y optimizar algunas interpretaciones de las narraciones que podrían contribuir al esclarecimiento de esta propuesta. Por ello, sería conveniente que en futuras investigaciones se complemente este trabajo cualitativo con entrevistas profundas que permitan esclarecer cualquier duda en la lectura de las biografías y reafirmar los presentes hallazgos.

\section{REFERENCIAS BIBLIOGRÁFICAS}

Aebi, M. F., 2004, “Crítica de la Criminología crítica: Una lectura escéptica de Baratta”, en F. Pérez-Alvarez (ed.), Serta in Memoriam Alexandri Baratta (pp. 17-56). Salamanca: Ediciones Universidad de Salamanca.

Alloza, A., 2001, "En busca de las causas del crimen. Teorías y estudios sobre delincuencia y justicia penal en la España Moderna”, Espacio, Tiempo y Forma, Serie IV, 14, 473-489.

Allwood, M. A. et al., 2012, "Depressive symptoms, including lack of future orientation, as mediators in the relationship between adverse life events and delinquent behaviors", in Journal of Child \& Adolescent Trauma, 5(2), 114-128. doi: 10.1080/19361521.2012.671795.

Baumrind, D., 2005, "Patterns of parental authority and adolescent autonomy", in New Directions for Child and Adolescent Development, (108), 61-69. doi: 10.1002/cd.128.

Berti, C. y Pivetti, M., 2019, "Childhood economic disadvantage and antisocial behavior: Intervening factors and pathways", in Children and Youth Services Review. Elsevier, June, 2017 (97), 120-126. doi: 10.1016/j.childyouth.2017.06.007.

Caplan, J., 2006, "Parole system anomie: conflicting models of casework and surveillance", in Federal probation. A journal of correctional philosophy and practice, 70 (3), 32-64.

Chen, M. S. y Foshee, V. A., 2015, "Stressful Life Events and the Perpetration of Adolescent Dating Abuse", in Journal of Youth and Adolescence, 44(3), 696-707. doi: 10.1007/s10964-014-0181-0.

CIDAC, C. de I. para el D., 2016, Justicia para Adolescentes en México: ¿Se garantizan los derechos de los jóvenes? Disponible en http://cidac.org/wp-content/ uploads/2016/02/adolescentes-web.pdf.

Cornejo, M., Mendoza, F. y Rojas, R. C., 2008, “La Investigación con Relatos de Vida: Pistas y Opciones del Diseño Metodológico", in Psykhe (Santiago), 17(1), 29-39. doi: 10.4067/s0718-22282008000100004. 
Cracco, C. y Blanco Larrieux, M. L., 2015. "Estresores y estrategias de afrontamiento en familias en las primeras etapas del ciclo vital y contexto socioeconómico", en Ciencias Psicológicas, 9(2), 129-140.

Crossley, M. L., 2002, "Introducing narrative psychology", in Psychology, 2002, 1-14. doi: $10.1177 / 135910530100600511$.

DeKeseredy, W., y Dragiewicz, M, 2018, Routledge handbook of critical criminology, London: Routledge. Taylor \& Francis Group. doi.org/10.4324/9781315622040

Eskin, M. et al., 2014, "Social problem-solving, perceived stress, negative life events, depression and life satisfaction in psoriasis", in Journal of the European Academy of Dermatology and Venereology, 28(11), 1553-1559. doi: 10.1111/ jdv. 12355 .

Estrada-Pineda, C. et al., 2015, "Parental implications in criminal behavior of teenagers: Trends and narratives", en Papeles de Poblacion, 21(84), 107-132.

Fanti, K. A., Colins, O. F. y Andershed, H., 2019, "Unraveling the longitudinal reciprocal associations between anxiety, delinquency, and depression from early to middle adolescence", in Journal of Criminal Justice, (62), 29-34. doi: 10.1016/j. jcrimjus.2018.09.004.

Farrington, D. P., 2002, “Criminology”, in Criminal Behavior and Mental Health, $12(4), 510-516$.

Freixa, E., 1978, "Reflexiones críticas acerca de la transposición del modelo médico a la psicología: su repercusión sobre los peritajes médico-legales en psiquiatría”, en Anuario de Sociología y Psicología Juridícas, 5, 31-40.

Goodley, D., 2001, “Learning difficulties', the social model of disability and impairment: challenging epistemologies", in Disability \& Society, 2(16), 207-231. doi: http://dx.doi.org/10.1080/09687590120035816.

Grover, K. et al., 2009, "Problem Solving Moderates the Effects of Life Event Stress and Chronic Stress on Suicidal Behaviors in Adolescence", in Journal of Clinical Psychology, 65(12), 1281-1290. doi: 10.1002/jclp.20632.

Guillén, A. I. et al., 2015, "Suicide attempts and stressful life events among female victims of intimate partner violence living in poverty in Nicaragua", in Scandinavian Journal of Psychology, 56(3), 349-356. doi: 10.1111/sjop.12207.

Herrero, J. et al., 2016, "Generalists versus specialists: Toward a typology of batterers in prison", in European Journal of Psychology Applied to Legal Context. Colegio Oficial de Psicólogos de Madrid, 8(1), 19-26. doi: 10.1016/j. ejpal.2015.09.002.

Hoeber, L. y Shaw, S., 2017, “Contemporary qualitative research methods in sport management", in Sport Management Review. Sport Management Association of Australia and New Zealand, 20(1), 4-7. doi: 10.1016/j.smr.2016.11.005.

Hoffmann, J. P., 2003, “A Contextual Analysis of Differential Association, Social Control, and Strain Theories of A Contextual Analysis of Differential Associa- 
tion, Social Control, and Strain Theories of Delinquency", in Social Forces, 81(3), 753-785. doi: 10.1353/sof.2003.0034.

Jaiswal, S. V et al., 2016, "Stressful life events, hopelessness, and suicidal intent in patients admitted with attempted suicide in a tertiary care general hospital", in Journal of postgraduate medicine, 62(2), 102-4. doi: 10.4103/0022-3859.180556.

Jennings, W. G. et al., 2019, "The linkage between mental health, delinquency, and trajectories of delinquency: results from the Boricua Youth Study", in Journal of Criminal Justice. Elsevier, 62(August 2018), pp. 66-73. doi: 10.1016/j.jcrimjus.2018.08.003.

Jiménez, L., Menéndez, S. e Hidalgo, M. V., 2008, “Un análisis de los acontecimientos vitales estresantes durante la adolescencia", en Apuntes de Psicología, 26(2008), 427-440.

Josselson, R., 2006, "Narrative research and the challenge of accumulating knowledge", in Narrative Inquiry, 16(1), 3-10.

Kim, J. H. et al., 2014, "Childhood maltreatment, stressful life events, and alcohol craving in adult drinkers", in Alcoholism: Clinical and Experimental Research, 38(7), 2048-2055. doi: 10.1111/acer.12473.

Kulkarni, P. N. y Patwardhan, V., 2015, "Stressful life events and irrational beliefs as predictors of Psychological Wellbeing among early adolescent girls in Pune, India", in Gender \& Behaviour, 13(1), 6507-6514. Disponible en http://search. ebscohost.com/login.aspx?direct $=$ true $\& d b=$ psyh \&AN=2015-29540-005\&site $=$ ehost-live\%5Cnhttp://vanita.patwardhan@jnanaprabodhini.org\%5Cnhttp://pradnyanitin05@yahoo.com.

Leppink, J., 2017, "Revisiting the quantitative-qualitative-mixed methods labels: Research questions, developments, and the need for replication", in Journal of Taibah University Medical Sciences. Elsevier Ltd, 12(2), 97-101. doi: 10.1016/j. jtumed.2016.11.008.

Lieblich, A., Tuval-Mashiach, R. y Zilber, T., 1998, Narrative Research: Reading, Analysis, and Interpretation. Editado por Sage. Thousand Oaks, CA.

Lila, M., Gracia, E. and Murgui, S., 2013, "Psychological adjustment and victim-blaming among intimate partner violence offenders: The role of social support and stressful life events", in The European Journal of Psychology Applied to Legal Context 5, 147-153. http://dx.doi.org/10.5093/ejpalc2013a4

Lippert, R. and Hamilton, C., 2020, “Governing Through Human Rights and Critical Criminology", in Critical Criminology, 28, 5-11. https://doi.org/10.1007/ s10612-020-09501-9

Maschi, T., 2006, "Unraveling the link between trauma and male delinquency: the cumulative versus differential risk perspectives.", in Social work, 51(1), 59-70. doi: $10.1093 / \mathrm{sw} / 51.1 .59$.

Maschi, T. y Bradley, C., 2008, "Exploring the moderating influence of delinquent peers on the link between trauma, anger, and violence among Male Youth: Impli- 
cations for social work practice", in Child and Adolescent Social Work Journal, 25(2), 125-138. doi: 10.1007/s10560-008-0116-2.

Mcleer, J. y Dehart, D., 2013, “Childhood Instability and Girls’ Delinquency: Role of Changes in Schools, Homes, and Caregivers", in Journal of Child \& Adolescent Trauma, 6(c), 74-89. doi: 10.1080/19361521.2013.735353.

Meo, A. y Navarro, A., 2009, “Enseñando a hacer entrevistas en investigación cualitativa: entre el oficio, la profesión y el arte", en Empiria. Revista de metodología de ciencias sociales, (17), 123-140. doi: 10.5944/empiria.17.2009.1990.

Oliva, A. et al., 2008, “Acontecimientos Vitales Estresantes, Resiliencia Y Ajuste Adolescente", en Revista de Psicopatología y Psicología Clínica, 13(1), 53-62. doi: 10.5944/rppc.vol.13.num.1.2008.4050.

Peleg, O., 2014, "The relationships between stressful life events during childhood and differentiation of self and intergenerational triangulation in adulthood", in International Journal of Psychology, 49(6), 462-470. doi: 10.1002/ijop.12054.

Phillips, A. C., Carroll, D. y Der, G., 2015, "Negative life events and symptoms of depression and anxiety: stress causation and/or stress generation", in Anxiety, Stress, \& Coping, 28(4), 357-371. doi: 10.1080/10615806.2015.1005078.

Rey-Bruguera, M., Calonge-Romano, I. and Martínez-Arias, M. R., 2017, "Los acontecimientos vitales estresantes, la sintomatología y la adaptación en la infancia: estudio comparativo con pacientes de salud mental y escolares", en Clínica y Salud, 28, 123-129. http://dx.doi.org/10.1016/j.clysa.2017.05.005

Rubens, S. L. et al., 2013, “Examining relations between negative life events, time spent in the United States, language use, and mental health outcomes in Latino adolescents", in Child \& Youth Care Forum, 42(5), 389-402. doi: http://dx.doi. org/10.1007/s10566-013-9205-2.

Samanci, O., 2010, “Teacher views on social skills development in primary school students", in Education, 131(1), 147-157.

Sanz Rodríguez, L. J. et al., 2009, "Estructura familiar, acontecimientos vitales estresantes y psicopatología en la adolescencia", in Family structure, stressful life events and psychopathology in the teenager, 29(104), 501-521. doi: 10.4321/ S0211-57352009000200015

Singh, A. P. y Dubey, A. P., 2015, Role of social support as a mediator in negative stressful life event and somatic complaints of managerial personnel, 6(3), 249-254.

Sokratous, S. et al., 2013, “The association between stressful life events and depressive symptoms among Cypriot university students: a cross-sectional descriptive correlational study", in BMC Public Health, 13(1), 1121. doi: 10.1186/14712458-13-1121.

Suarez, Á. M., 2010, "La importancia del análisis de los acontecimientos vitales estresantes en la práctica clínica”, en Artículos de Revisión Rev Med La Paz, 16(2), 58-62. 
Tamers, S. L. et al., 2014, "The impact of stressful life events on excessive alcohol consumption in the French population: Findings from the GAZEL cohort study", in PLoS ONE, 9(1). doi: 10.1371/journal.pone.0087653.

Torío, S., Peña, J. V. y Rodríguez, M. C., 2008, "Estilos educativos parentales. Revisión bibliográfica y reformulación teórica", en Teor. educ. 20, 151-178

Zepeda, G., 2004, Crimen sin castigo. Procuración e justicia penal y ministerio público en México. México: Fondo de Cultura Económica.

Vázquez, C., 2003, Delincuencia juvenil. Consideraciones penales y criminologías. Madrid: Colex,

Zolog, T. C. et al., 2011, "Edad, género y acontecimientos vitales estresantes en autoinformes de ansiedad y depresión en preado-lescencia y adolescencia temprana", en Ansiedad y Estrés, 17(2-3), 113-124.

\section{RESUMEN CURRICULAR DE LOS AUTORES}

\section{Cristina Estrada Pineda}

Profesora Investigadora del Departamento de Trabajo Social del Centro Universitario de Ciencias Sociales y Humanidades de la Universidad de Guadalajara, México. Es Maestra en Terapia Familiar por la Universidad de Guadalajara y Doctora por la Facultad de Psicología de la Universidad de Oviedo, España. Se desempeñó como terapeuta familiar en la Comisaría de Justicia para Adolescentes. Actualmente ejerce como Profesora Investigadora del Departamento de Trabajo Social, con una trayectoria de más de 24 años de servicio. Desde 2009 es miembro del Sistema Nacional de Investigadores, actualmente en el Nivel II. Las líneas de investigación que desarrolla se dirigen al estudio de la delincuencia, la violencia en las relaciones de pareja, la familia y el apoyo social. Ha desarrollado diferentes investigaciones con y sin financiamiento tanto a nivel nacional como internacional.

Dirección electrónica: cristina.estrada@csh.udg.mx

Registro ORCID: https://orcid.org/0000-0002-8699-2666

\section{Francisco Javier Rodríguez Díaz}

Catedrático de Personalidad, Evaluación y Tratamiento Psicológico. Departamento de Psicología de la Universidad de Oviedo, España. Es Licenciado en Filosofía y Ciencias de la Educación, Sección de Psicología, por la Universidad de Santiago de Compostela. Defendió su Tesis Doctoral en la misma universidad, en 1986. En la actualidad es Catedrático de Universidad del Departamento de Psicología de la Universidad de Oviedo, contando con una evaluación positiva de cuatro sexenios de investigación 
desde 1987. La trayectoria científica se adscribe a la línea de investigación en torno a la Psicología Jurídica y Forense (Violencia, Menor Infractor, Comportamiento Antisocial, y Violencia Doméstica), que han tenido tanto continuidad en la financiación de $\mathrm{I}+\mathrm{D}+\mathrm{i}$ como en la producción científica de calidad, dentro del grupo de investigación Psicología Comunitaria, Jurídica y Salud (CJS).

Dirección electrónica: gallego@uniovi.es

Registro ORCID: https://orcid.org/0000-0002-5899-439X

\section{Carolina Bringas Molleda}

Profesora Contratada Doctora. Departamento de Psicología y Antropología de la Universidad de Extremadura, España. Licenciada y Doctora en Psicología por la Universidad de Oviedo. Actualmente es Profesora Contratada Doctora del Departamento de Psicología y Antropología de la Universidad de Extremadura, dentro del área Psicología Evolutiva y de la Educación. Ha publicado diversos artículos en revistas científicas, y capítulos de libro, así como contribuido a diversos congresos nacionales e internacionales. También ha participado como miembro investigador en Proyectos de Investigación financiados por diversos organismos. Actualmente cuenta con un sexenio de investigación.

Dirección electrónica: cbringas@unex.es

Registro ORCID: https://orcid.org/0000-0001-7956-2337

\section{Mario Alberto Morales Martínez}

Profesor Investigador del Departamento de Cultura, Justicia y Democracia. Centro Universitario del Norte de la Universidad de Guadalajara. Es Maestro y Doctor en Ciencias Sociales por la Universidad de Guadalajara, de donde también egresó como Licenciado en Sociología. Actualmente se desempeña como Profesor Investigador de Tiempo Completo en el Centro Universitario del Norte de la Universidad de Guadalajara. En su haber cuenta con diversas publicaciones en libros especializados y revistas científicas; así como también ha contribuido a diversos congresos nacionales e internacionales. Ha participado como responsable y colaborador en diversos proyectos de investigación con y sin financiamiento en la línea de estudios de bienestar, políticas sociales y sociedad. Sus actuales proyectos de investigación tratan diferentes problemáticas psicosociales y educativas con un especial énfasis en la situación de los jóvenes en México.

Dirección electrónica: mario.mmartinez@academicos.udg.mx Registro ORCID: https://orcid.org/0000-0003-0866-9756

Artículo recibido el 01 de agosto de 2019 y aprobado el 29 de agosto de 2020. 